\title{
Concise Total Synthesis of (+)-Aphanorphine
}

\author{
Cheng Wang \\ Yukun Guan* \\ School of Pharmacy, Yantai University, Qingquan Road-30, \\ Yantai 264005, P. R. of China \\ gyk@ytu.edu.cn
}

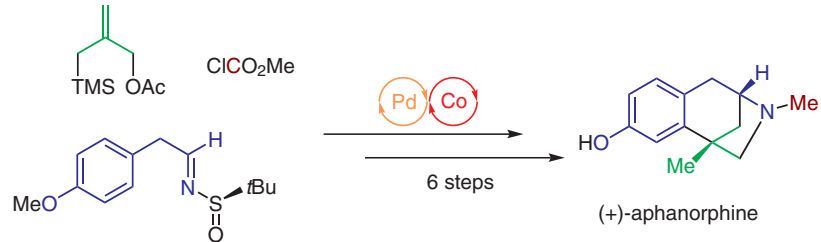

tion of the 2-benzylpyrrolidine intermediate to construct the ring $B,{ }^{2}$ transannular enolate or radical cyclization of 3benzazepine derivatives to form both rings $\mathrm{B}$ and $\mathrm{C},{ }^{3}$ or intramolecular nucleophilic cyclization of tetralin or dihydronaphthalene substrates to build ring C. ${ }^{4}$ Grainger developed a unique approach including a carbamoyl-radical cyclization to close ring $C$ and a late-stage formation of aromatic ring A through an inverse-electron-demand DielsAlder reaction. ${ }^{5}$ Here, we report a concise total synthesis of (+)-aphanorphine (5) based on transition metal-catalyzed cyclization reactions.

The metal-catalyzed hydrogen-atom transfer (MHAT) reaction has emerged as a powerful tool in organic synthesis. ${ }^{6,7}$ As shown in Scheme 1, we envisioned that the ring B and $\mathrm{C} 1$ quaternary carbon center of (+)-aphanorphine (5) might be obtained by a radical cyclization initiated by MHAT of the 2-benzylpyrrolidine $\mathbf{6}$, which, in turn, could be assembled by intermolecular trimethylenemethane (TMM) $[3+2]$-cycloaddition ${ }^{8}$ of the known chiral imine 7 with 2[(trimethylsilyl)methyl]allyl acetate (8) (Scheme 1).

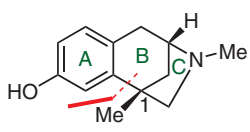

(+)-aphanorphine (5)

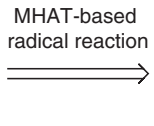

TMM [3+2] $\mathrm{TMM}[3+2]$
cycloaddition cycloaddition
Scheme 1 Retrosynthetic analysis of (+)-aphanorphine (5)

Our total synthesis of $(+)$-aphanorphine $(\mathbf{5})$ commenced with the TMM [3+2]-cycloaddition of 2-[(trimethylsilyl)methyl]allyl acetate (8) with the chiral imine $\mathbf{7}$ (Scheme $2),{ }^{9}$ which can be prepared from (4-methoxyphenyl)acetaldehyde $(\mathbf{9})$ and $(R)-(+)$-tert-butylsulfinamide (10) in 66\% yield by a known procedure. ${ }^{10}$ Stockman and co-workers

Figure 1 Representative benzomorphan alkaloids 
previously investigated the TMM [3+2]-cycloadditions of chiral aryl and alkyl tert-butanesulfinimines to yield enantiopure pyrrolidine products. ${ }^{11}$ Unfortunately, when we followed Stockman's method, none of the desired cycloaddition product was detected when $\mathbf{7}$ and $\mathbf{8}$ were stirred with $\operatorname{Pd}\left(\mathrm{PPh}_{3}\right)_{4}$ in THF for 18 hours at $25^{\circ} \mathrm{C}$. Instead, the unexpected alkylation product 11 was isolated in $42 \%$ yield (Scheme 2a). We surmised that $\mathbf{1 1}$ might be formed by proton transfer from the $\mathrm{C} 5$ atom of $\mathbf{7}$ to the Pd-TMM intermediate 12 . The $\mathrm{C} 5$ position of $\mathbf{7}$ is activated by both an electron-withdrawing inductive effect of the imine group and by the conjugate effect of the phenyl group; consequently, instead of the expected cycloaddition of the TMM intermediate 12 with the imine, proton transfer from the $\mathrm{C} 5$ atom of $\mathbf{7}$ to the Pd-TMM intermediate $\mathbf{1 2}$ becomes the favored pathway to give methallyl complex $\mathbf{1 3}$, which is attacked by the resulting anion $\mathbf{1 4}$ to deliver the alkylation product $\mathbf{1 1} .^{12}$
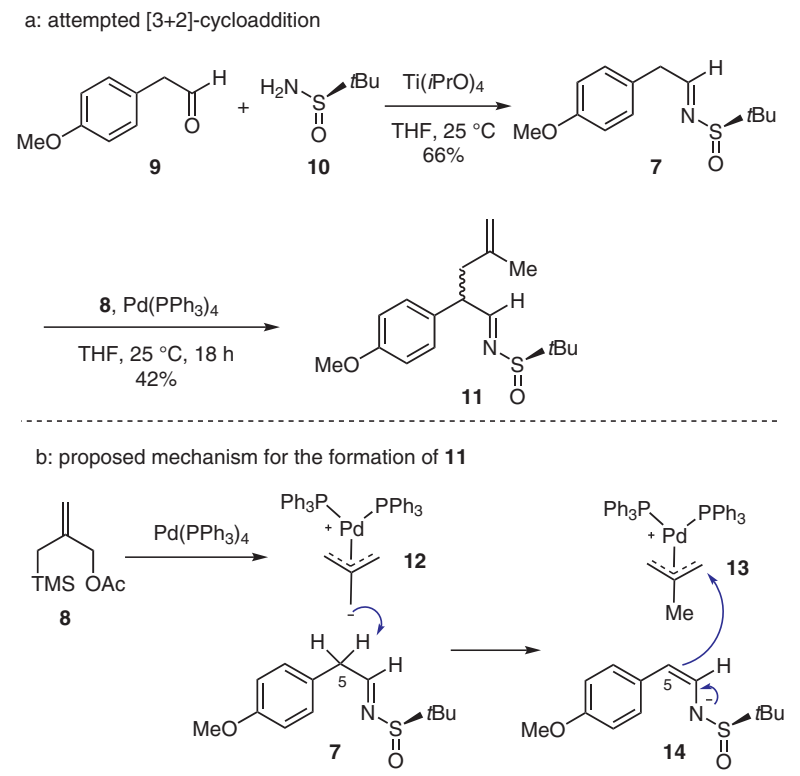

$\longrightarrow 11$

Scheme 2 Investigation of the [3+2]-cycloaddition

Reports by Trost and co-workers ${ }^{12 a, c}$ suggested that increasing the temperature might enhance the nucleophilicity of TMM. Pleasingly, when the reaction mixture was stirred under reflux for 19 hours, our desired cycloaddition products 15a and 15b were obtained in $1: 3 \mathrm{dr}$ with a combined yield of $52 \%$, along with the mono- and dialkylation products 11 and 16, respectively, in yields of 9 and 19\%. For the synthesis of (+)-aphanorphine (5), the tert-butylsulfinyl group of 15b was removed by treatment with $2 \mathrm{M} \mathrm{HCl}$ in $\mathrm{MeOH}$, and the resulting secondary amine was treated with $\mathrm{ClCO}_{2} \mathrm{Me}$ in the presence of $\mathrm{NEt}_{3}$ to give the methyl carbamate $\mathbf{1 7}$ in $80 \%$ yield over the two steps (Scheme 3).
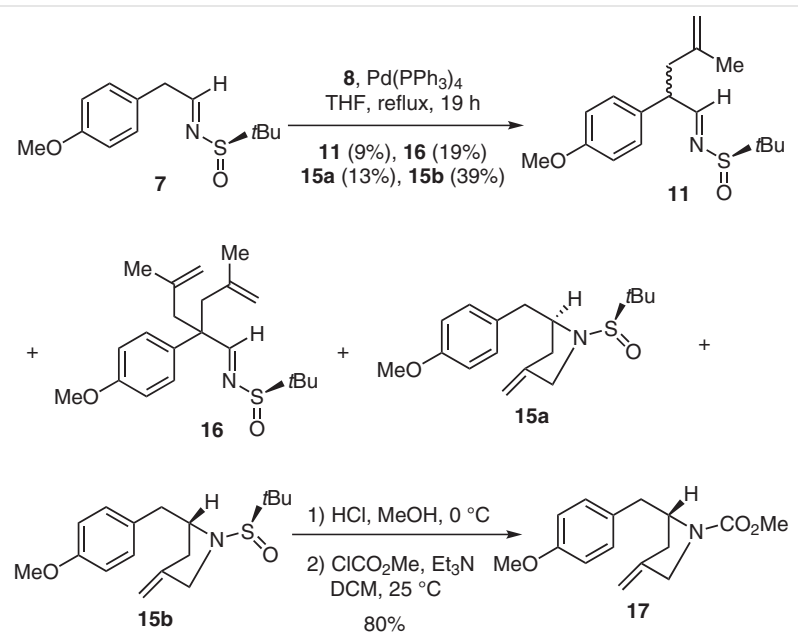

Scheme 3 Synthesis of benzylpyrrolidine 17

According to our synthetic plan, the next work was to construct the tricyclic benzazepine core of (+)-aphanorphine (5) through MHAT-based radical cycloaddition. We began our study by evaluating a catalytic system previously used by Shigehisa et al. for the hydroarylation of nonactivated alkenes (Table 1 ). ${ }^{13}$ Treatment of $\mathbf{1 7}$ with $1,1,3,3$-tetramethyldisiloxane (TMDSO), $\mathrm{N}$-fluoro-2,4,6-trimethylpyridinium triflate (01, Figure 2), and the ethylenediamine-containing salen Co-catalyst $\mathbf{C 1}$ in $\mathrm{PhCF}_{3}$ gave the desired tricyclic benzazepine $\mathbf{1 8}$ in only $6 \%$ yield (Table 1 , entry 1 ). To our delight, the use of the 1,3-diaminopropanecontaining catalyst C2 (Figure 2) improved the yield to $72 \%$ (entry 2). The longer 1,4-butanediamine gave a much lower yield (entry 3). Replacing the tert-butyl group on the 5-position of the aromatic ring of $\mathbf{C 2}$ with $\mathrm{H}$, Me, or OMe (C4C6) led to no conversion (entries 4-6). Further catalyst screening showed that $\mathbf{C 7}$ was the best catalyst, affording a $76 \%$ yield of the desired product (entries 7 and 8 ). Next, a series of oxidants including $\mathrm{N}$-fluoro-2,4,6-trimethylpyridinium tetrafluoroborate $(\mathbf{O 2}), \mathrm{N}$-fluoropyridinium triflate (03), $\mathrm{N}$-fluoropyridinium tetrafluoroborate (04), and (diacetoxyiodo)benzene (05) were evaluated, but all proved inferior to $\mathrm{N}$-fluoro-2,4,6-trimethylpyridinium triflate (01) (entries 9-12). Finally, we examined various silanes and we found that poly(methylhydrosiloxane)(PMHS) was superior to TMDSO, $\mathrm{PhSiH}_{3}$, or $\mathrm{Ph}(i-\mathrm{PrO}) \mathrm{SiH}_{2},{ }^{14}$ giving an improved yield of $83 \%{ }^{15}$ (entries $13-15$ ).

With 18 in hand, the remaining transformations of the synthesis were $\mathrm{N}$-methylation and O-demethylation. Reduction of 18 with excess $\mathrm{LiAlH}_{4}$ afforded (-)-8-O-methylaphanorphine (19) in $88 \%$ yield. On following the procedure of Fuchs and Funk, ${ }^{3 a}$ treatment of 19 with $\mathrm{BBr}_{3}$ in DCM at a low temperature effected the expected O-demethylation, giving (+)-aphanorphine (5) in 50\% yield (Scheme 4 ). 
The physical and spectroscopic data of the synthetic (+)aphanorphine (5) $\left\{[\alpha]_{D}{ }^{25}+20.8(c 0.4, \mathrm{MeOH})\right\}$ agreed with those reported previously. ${ }^{1,21}$

Table 1 Optimization of the MHAT-Based Radical Cycloaddition

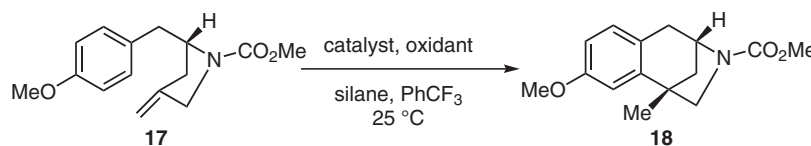

\begin{tabular}{|c|c|c|c|c|}
\hline Entry & Catalyst $^{\mathrm{a}}$ & Silane & Oxidant $^{\mathrm{a}}$ & Yield $^{\mathrm{b}}(\%)$ \\
\hline 1 & C1 & TMDSO & 01 & 6 \\
\hline 2 & $\mathrm{C} 2$ & TMDSO & 01 & 72 \\
\hline 3 & C3 & TMDSO & 01 & 12 \\
\hline 4 & C4 & TMDSO & 01 & $N^{c}$ \\
\hline 5 & C5 & TMDSO & 01 & ND \\
\hline 6 & C6 & TMDSO & 01 & ND \\
\hline 7 & C7 & TMDSO & 01 & 76 \\
\hline 8 & C8 & TMDSO & 01 & 13 \\
\hline 9 & C7 & TMDSO & 02 & 58 \\
\hline 10 & C7 & TMDSO & 03 & ND \\
\hline 11 & C7 & TMDSO & 04 & 36 \\
\hline 12 & C7 & TMDSO & 05 & 38 \\
\hline 13 & C7 & $\mathrm{PhSiH}_{3}$ & 01 & 32 \\
\hline 14 & C7 & PMHS & 01 & 83 \\
\hline 15 & C7 & $\mathrm{PhSiH}_{2}(\mathrm{O}-i-\mathrm{Pr})$ & 01 & 27 \\
\hline
\end{tabular}

${ }^{a}$ For catalyst and oxidant structures, see Figure 2.

b Isolated yield.

${ }^{\mathrm{C}} \mathrm{ND}=$ not detected.
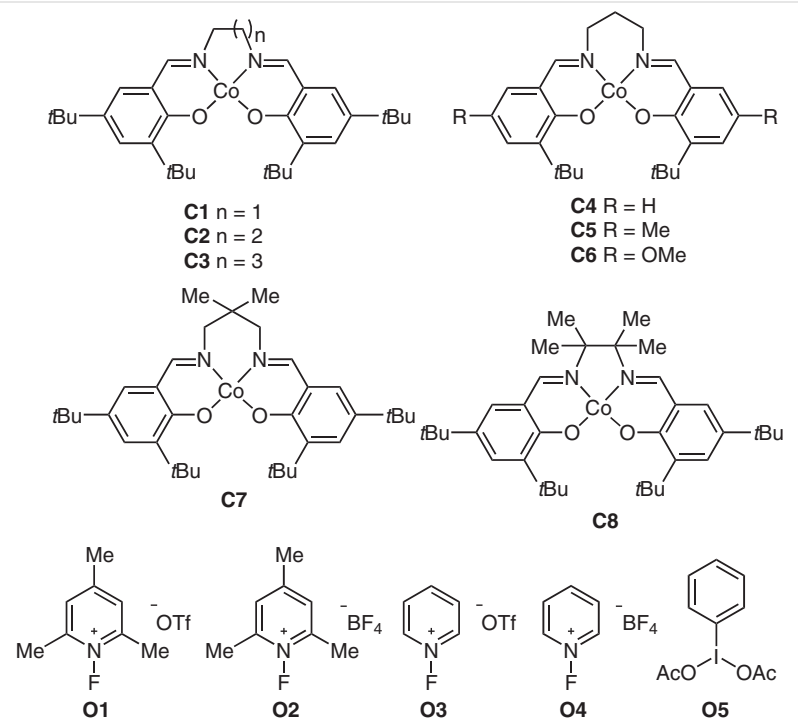

Figure 2 Catalyst structures $\mathrm{C} 1-\mathrm{C} 8$ and oxidants 01-05
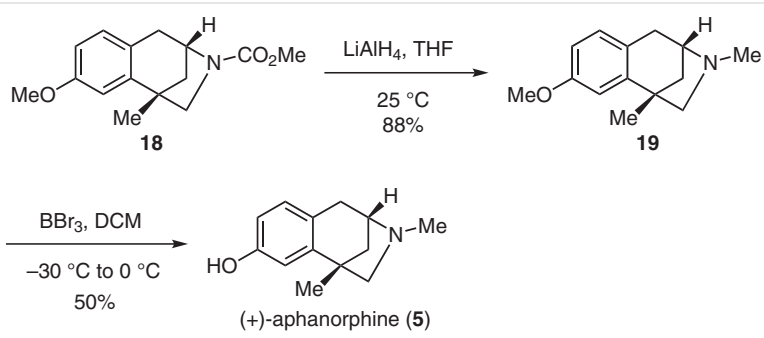

Scheme 4 Completion of the total synthesis of (+)-aphanorphine (5)

In summary, a concise total synthesis of (+)-aphanorphine (5) was achieved, starting from the known chiral tertbutanesulfinimine $\mathbf{7}$, in six steps and $11 \%$ overall yield. The transition-metal-catalyzed intermolecular TMM [3+2]-cycloaddition and a MHAT-based radical cyclization were used in a rapid construction of the tricyclic benzazepine core of the natural product. In addition, methyl carbamate was used as a latent methylamine, avoiding additional steps involving manipulation of $\mathrm{N}$-substituent group, as required in the previous synthesis, thereby improving the overall synthetic efficiency.

\section{Conflict of Interest}

The authors declare no conflict of interest.

\section{Funding Information}

This work was financially supported by the Natural Science Foundation of Shandong Province (ZR2018PB006).

\section{Acknowledgment}

We thank Prof. Chun-An Fan (Lanzhou University) for assistance in measuring the optical rotations.

\section{Supporting Information}

Supporting information for this article is available online at https://doi.org/10.1055/s-0037-1610769.

\section{References and Notes}

(1) Gulavita, N.; Hori, A.; Shimizu, Y.; Laszlo, P.; Clardy, J. Tetrahedron Lett. 1988, 29, 4381.

(2) (a) Tamura, O.; Yanagimachi, T.; Kobayashi, T.; Ishibashi, H. Org. Lett. 2001, 3, 2427. (b) Zhai, H.; Luo, S.; Ye, C.; Ma, Y. J. Org. Chem. 2003, 68, 8268. (c) Hu, H.; Zhai, H. Synlett 2003, 2129. (d) Tamura, O.; Yanagimachi, T.; Ishibashi, H. Tetrahedron: Asymmetry 2003, 14, 3033. (e) Bower, J. F.; Szeto, P.; Gallagher, T. Chem. Commun. 2005, 5793. (f) Bower, J. F.; Szeto, P.; Gallagher, T. Org. Biomol. Chem. 2007, 5, 143. (g) Ma, Z.; Zhai, H. Synlett 2007, 161. (h) Ma, Z.; Hu, H.; Xiong, W.; Zhai, H. Tetrahedron 2007, 63, 7523. (i) Yang, X.; Zhai, H.; Li, Z. Org. Lett. 2008, 10, 2457. (j) Yang, X.; Cheng, B.; Li, Z.; Zhai, H. Synlett 2008, 
2821. (k) Yoshimitsu, T.; Atsumi, C.; Iimori, E.; Nagaoka, H.; Tanaka, T. Tetrahedron Lett. 2008, 49, 4473. (1) Mai, D. N.; Rosen, B. R.; Wolfe, J. P. Org. Lett. 2011, 13, 2932. (m) Medjahdi, M.; González-Gómez, J. C.; Foubelo, F.; Yus, M. Eur. J. Org. Chem. 2011, 2230. (n) Pansare, S. V.; Kulkarni, K. G. RSC Adv. 2013, 3, 19127. (o) Wang, Z.; Zheng, H.; Yang, J.; Xie, X.; She, X. Adv. Synth. Catal. 2015, 357, 2082. (p) Peterson, L. J.; Wolfe, J. P. Adv. Synth. Catal. 2015, 357, 2339.

(3) (a) Fuchs, J. R.; Funk, R. L. Org. Lett. 2001, 3, 3923. (b) Katoh, M.; Inoue, H.; Suzuki, A.; Honda, T. Synlett 2005, 2820. (c) Honda, T.; Katoh, M.; Inoue, H. Heterocycles 2007, 72, 497. (d) Donets, P. A.; Goeman, J. L.; Van der Eycken, J.; Robeyns, K.; Van Meervelt, L.; Van der Eycken, E. V. Eur. J. Org. Chem. 2009, 793.

(4) (a) Takano, S.; Inomata, K.; Sato, T.; Ogasawara, K. J. Chem. Soc., Chem. Commun. 1989, 1591. (b) Takano, S.; Inomata, K.; Sato, T.; Takahashi, M.; Ogasawara, K. J. Chem. Soc., Chem. Commun. 1990, 290. (c) Honda, T.; Yamamoto, A.; Cui, Y.; Tsubuki, M. J. Chem. Soc., Perkin Trans. 1 1992, 531. (d) Hulme, A. N.; Henry, S. S.; Meyers, A. I. J. Org. Chem. 1995, 60, 1265. (e) Fadel, A.; Arzel, P. Tetrahedron: Asymmetry 1995, 6, 893. (f) Hallinan, K. O.; Honda, T. Tetrahedron 1995, 51, 12211. (g) Meyers, A. I.; Schmidt, W.; Santiago, B. Heterocycles 1995, 40, 525. (h) Shiotani, S.; Okada, H.; Nakamata, K.; Yamamoto, T.; Sekino, F. Heterocycles 1996, 43, 1031. (i) Node, M.; Imazato, H.; Kurosaki, R.; Kawano, Y.; Inoue, T.; Nishide, K.; Fuji, K. Heterocycles 1996, 42, 811. (j) Ogasawara, K.; Shimizu, M.; Kamikubo, T. Heterocycles 1997, 46, 21. (k) Fadel, A.; Arzel, P. Tetrahedron: Asymmetry 1997, 8, 283. (l) Fadel, A.; Arzel, P. Tetrahedron: Asymmetry 1997, 8, 371. (m) Tanaka, K.; Taniguchi, T.; Ogasawara, K. Tetrahedron Lett. 2001, 42, 1049. (n) ElAzab, A. S.; Taniguchi, T.; Ogasawara, K. Heterocycles 2002, 56, 39. (o) Kita, Y.; Futamura, J.; Ohba, Y.; Sawama, Y.; Ganesh, J. K.; Fujioka, H. J. Org. Chem. 2003, 68, 5917. (p) Taylor, S. K.; Ivanovic, M.; Simons, L. J.; Davis, M. M. Tetrahedron: Asymmetry 2003, 14, 743. (q) Li, M.; Zhou, P.; Roth, H. F. Synthesis 2007, 55. (r) Zhu, D.-Y.; Xu, M.-H.; Tu, Y.-Q.; Zhang, F.-M.; Wang, S.-H. Chem. Eur. J. 2015, 21, 15502. (s) Chiou, W.-H.; Chen, P.-C. J. Org. Chem. 2017, $82,8213$.

(5) Grainger, R. S.; Welsh, E. J. Angew. Chem. Int. Ed. 2007, 46, 5377.

(6) For reviews about MHAT reaction, see: (a) Crossley, S. W. M.; Obradors, C.; Martinez, R. M.; Shenvi, R. A. Chem. Rev. 2016, 116, 8912. (b) Green, S. A.; Crossley, S. W. M.; Matos, J. L. M.; Vásquez-Céspedes, S.; Shevick, S. L.; Shenvi, R. A. Acc. Chem. Res. 2018, 51, 2628.

(7) For selected applications MHAT reaction in natural product synthesis, see: (a) Zhang, B.; Zheng, W.; Wang, X.; Sun, D.; Li, C. Angew. Chem. Int. Ed. 2016, 55, 10435. (b) Xu, G.; Elkin, M.; Tantillo, D. J.; Newhouse, T. R.; Maimone, T. J. Angew. Chem. Int. Ed. 2017, 56, 12498. (c) Deng, H.; Cao, W.; Liu, R.; Zhang, Y.; Liu, B. Angew. Chem. Int. Ed. 2017, 56, 5849. (d) Godfrey, N. A.; Schatz, D. J.; Pronin, S. V. J. Am. Chem. Soc. 2018, 140, 12770. (e) Lu, Z.; Zhang, X.; Guo, Z.; Chen, Y.; Mu, T.; Li, A. J. Am. Chem. Soc. 2018, 140, 9211. (f) Farney, E. P.; Feng, S. S.; Schäfers, F.; Reisman, S. E. J. Am. Chem. Soc. 2018, 140, 1267. (g) Ji, Y.; Xin, Z.;
He, H.; Gao, S. J. Am. Chem. Soc. 2019, 141, 16208. (h) Xu, G.; Wu, J.; Li, L.; Lu, Y.; Li, C. J. Am. Chem. Soc. 2020, 142, 15240. (i) Chen, P.; Wang, C.; Yang, R.; Xu, H.; Wu, J.; Jiang, H.; Chen, K.; Ma, Z. Angew. Chem. Int. Ed. 2021, 60, 5512.

(8) Yamago, S.; Nakamura, E. Org. React. (Hoboken, NJ, U. S.) 2002, $61,1$.

(9) For selected applications of $N$-tert-butanesulfinimines in natural-product synthesis, see: (a) Chuang, K. V.; Navarro, R.; Reisman, S. E. Angew. Chem. Int. Ed. 2011, 50, 9447. (b) Zhao, S.; Andrade, R. B. J. Am. Chem. Soc. 2013, 135, 13334. (c) Chogii, I.; Njardarson, J. T. Angew. Chem. Int. Ed. 2015, 54, 13706. (d) Tian, M.; Yan, M.; Baran, P. S. J. Am. Chem. Soc. 2016, 138, 14234. (e) Hugelshofer, C. L.; Palani, V.; Sarpong, R. J. Am. Chem. Soc. 2019, 141, 8431. (f) Li, Y.; Wang, C.; Ma, Z.; Zhang, K.; Xu, X.-T. Org. Lett. 2020, 22, 8589.

(10) Yao, Q.; Yuan, C. J. Org. Chem. 2013, 78, 6962.

(11) Procopiou, G.; Lewis, W.; Harbottle, G.; Stockman, R. A. Org. Lett. 2013, 15, 2030.

(12) (a) Trost, B. M.; Chan, D. M. T. J. Am. Chem. Soc. 1979, 101, 6432. (b) Trost, B. M.; Chan, D. M. T. J. Am. Chem. Soc. 1983, 105, 2326. (c) Trost, B. M.; Bringley, D. A.; O’Keefe, B. M. Org. Lett. 2013, 15, 5630.

(13) Shigehisa, H.; Ano, T.; Honma, H.; Ebisawa, K.; Hiroya, K. Org. Lett. 2016, 18, 3622.

(14) Obradors, C.; Martinez, R. M.; Shenvi, R. A. J. Am. Chem. Soc. 2016, $138,4962$.

(15) Methyl (1S,4S)-8-methoxy-1-methyl-1,2,4,5-tetrahydro-3H1,4-methanobenzo[d]azepine-3-carboxylate (18)

A $4 \mathrm{~mL}$ vial was charged with sulfinimine $17(13 \mathrm{mg}, 0.05$ mmol, 1.0 equiv), catalyst $\mathbf{C 7}$ ( $1.5 \mathrm{mg}, 0.0025 \mathrm{mmol}, 0.05$ equiv), and oxidant 01 (29 mg, $0.1 \mathrm{mmol}, 2.0$ equiv). $\mathrm{PhCF}_{3}(0.5 \mathrm{~mL}$ ), previously dried in vacuo for $0.5 \mathrm{~h}$, was then added and the solution was bubbled with $\mathrm{N}_{2}$ for $10 \mathrm{~min}$. PMHS $(22 \mu \mathrm{L}, 0.1$ mmol, 2.0 equiv) was added, and the resulting mixture was stirred at $25{ }^{\circ} \mathrm{C}$ for $20 \mathrm{~h}$ then diluted with EtOAc $(2 \mathrm{~mL})$. The solution was washed with $\mathrm{H}_{2} \mathrm{O}(0.5 \mathrm{~mL})$ and brine $(3 \times 0.5 \mathrm{~mL})$, then dried $\left(\mathrm{Na}_{2} \mathrm{SO}_{4}\right)$ and concentrated in vacuo. The residue was purified by preparative TLC (PE-EtOAc, 5:1) to give a yellow solid: yield; $10.8 \mathrm{mg}(83 \%) ; \mathrm{mp} 89-92{ }^{\circ} \mathrm{C} ;[\alpha]_{\mathrm{D}}^{25}+167.3$ (c 0.55 , $\mathrm{CHCl}_{3}$ ).

IR (KBr): 3795, 2957, 1701, 1612, 1495, 1453, 1389, 863, 805, $769,741,698 \mathrm{~cm}^{-1}$. Rotamer ${ }^{1} \mathrm{H}$ NMR $\left(500 \mathrm{MHz}, \mathrm{CDCl}_{3}\right): \delta=7.03$ $(\mathrm{d}, J=8.4 \mathrm{~Hz}, 0.5 \mathrm{H}), 6.99(\mathrm{~d}, J=8.3 \mathrm{~Hz}, 0.5 \mathrm{H}), 6.85-6.80(\mathrm{~m}, 1$ H), $6.72(\mathrm{td}, J=8.3,2.5 \mathrm{~Hz}, 1 \mathrm{H}), 4.50-4.43(\mathrm{~m}, 0.6 \mathrm{H}), 4.39-4.32$ (m, $0.4 \mathrm{H}), 3.82-3.74(\mathrm{~m}, 3 \mathrm{H}), 3.72-3.66(\mathrm{~m}, 1.3 \mathrm{H}), 3.63-3.58$ $(\mathrm{m}, 1.7 \mathrm{H}), 3.42(\mathrm{~d}, J=10.1 \mathrm{~Hz}, 0.5 \mathrm{H}), 3.36(\mathrm{~d}, J=9.9 \mathrm{~Hz}, 0.5 \mathrm{H})$, $3.28(\mathrm{~d}, J=10.0 \mathrm{~Hz}, 0.5 \mathrm{H}), 3.22(\mathrm{~d}, J=9.9 \mathrm{~Hz}, 0.5 \mathrm{H}), 3.18(\mathrm{~d}, J=$ $16.6 \mathrm{~Hz}, 0.5 \mathrm{H}), 3.04(\mathrm{~d}, J=16.6 \mathrm{~Hz}, 0.5 \mathrm{H}), 2.90(\mathrm{~d}, J=16.6 \mathrm{~Hz}, 1$ $\mathrm{H}), 2.02-1.86(\mathrm{~m}, 2 \mathrm{H}), 1.57-1.45(\mathrm{~m}, 3 \mathrm{H}) .{ }^{13} \mathrm{C}$ NMR $(125 \mathrm{MHz}$, $\left.\mathrm{CDCl}_{3}\right): \delta=157.9,157.8,155.1,154.9,145.8,145.7,130.5,130.3$, 125.7, 125.4, 111.6, 111.4, 109.89, 109.86, 61.6, 61.2, 55.29, 55.27, 54.8, 54.6, 52.2, 52.0, 42.2, 41.7, 41.6, 40.8, 36.4, 35.7, 20.8. HRMS (ESI): $\mathrm{m} / z[\mathrm{M}+\mathrm{H}]^{+}$calcd for $\mathrm{C}_{15} \mathrm{H}_{20} \mathrm{NO}_{3}: 262.1443$; found: 262.1437 . 